\title{
FUNCTORS INVOLVING C.S.S. COMPLEXES
}

\author{
BY \\ DANIEL M. KAN(')
}

1. Introduction. Using the theory of adjoint functors, developed in [4], a procedure will be given by which functors and natural transformations may be constructed which involve c.s.s. complexes. Several of the functors and natural transformations obtained in this manner are well known. A new such functor, $H^{v}(\Gamma$, ), from chain complexes to c.s.s. abelian groups, will be considered in more detail $\left({ }^{2}\right)$. It has the following properties

(a) The functor $H^{v}(\Gamma, \quad)$ sets up a one-to-one correspondence between chain complexes which are zero in dimension $<0$ and c.s.s. abelian groups.

(b) For every chain complex $K$

$$
H_{n}(K) \approx \pi_{n}\left(H^{v}(\Gamma, K),\right.
$$

i.e. the homology groups of the chain complex $K$ are isomorphic with the homotopy groups of the c.s.s. group $H^{v}(\Gamma, K)$.

(c) Let $(\pi, n)$ be a chain complex which has the abelian group $\pi$ in dimension $n$ and 0 in the others. Then

$$
H^{V}(\Gamma,(\pi, n))=K(\pi, n)
$$

i.e. $H^{v}(\Gamma,(\pi, n))$ is the Eilenberg-MacLane complex of $\pi$ on level $n$.

Two other functors obtained by the procedure mentioned above will be discussed in [5].

In an appendix we define for a c.s.s. complex $K$ the c.s.s. free abelian group $F A K$ generated by it. This notion is closely related to the functor $H^{v}(\Gamma, \quad)$. For every c.s.s. complex $K$

$$
H_{n}(K) \approx \pi_{n}(F A K)
$$$$
n>0 .
$$

There is a natural way of embedding $K$ into $F A K$. This embedding map $f: K \rightarrow F A K$ induces homomorphisms of the homotopy groups of $K$ into the homotopy groups of $F A K$ and hence by the above isomorphism into the homology groups of $K$. It will be shown that these homomorphisms are the Hurewicz homomorphisms.

The definitions and results of [4] will be used freely.

2. A definition of c.s.s. complexes. For each integer $n \geqq 0$ let $[n]$ denote the ordered set $(0, \cdots, n)$. By a monotone function $\alpha:[m] \rightarrow[n]$ we mean a function such that

Received by the editors September 20, 1956.

(1) The author is now at the Hebrew University in Jerusalem.

(2) This functor has also been found by A. Dold. 


$$
\alpha(i) \leqq \alpha(j) \quad 0 \leqq i \leqq j \leqq m .
$$

Clearly the composition of two monotone functions is again a monotone function and for every integer $n \geqq 0$ the identity map $\epsilon_{n}:[n] \rightarrow[n]$ is also monotone. Hence the ordered sets $[n]$ and the monotone functions $\alpha:[m] \rightarrow[n]$ form a (proper) category. Throughout this paper this category will be denoted by $v$.

Let $\mathfrak{T}$ be the category of sets. We recall that $\mathfrak{M}^{v}$ denotes the category of contravariant functors $\mho \rightarrow \mathfrak{N}$.

Definition (2.1). A c.s.s. complex $K$ is a contravariant functor $K: V \rightarrow \mathfrak{T}$, i.e. an object of the category $\mathfrak{T}^{V}$. Similarly a c.s.s. $\operatorname{map} f: K \rightarrow L$ is a natural transformation from $K$ to $L$, i.e. a map of the category $\mathfrak{N}^{v}$. The elements of the set $K[n]$ are called $n$-simplices of $K$.

The category of c.s.s. complexes and c.s.s. maps, i.e. the category $\mathfrak{T}^{v}$, will often be denoted by $S$.

It is readily verified that Definition (2.1) is equivalent with that of Eilenberg-Zilber $[2 ; 3]$, except that the collection of the $n$-simplices of a c.s.s. complex is required to be a set, i.e. an object of the category $\mathfrak{T}$. Following Eilenberg-Zilber we shall write $\sigma \alpha$ instead of $(K \alpha) \sigma$, where $\sigma \in K[n]$ and $\alpha:[m] \rightarrow[n]$ is a monotone function.

3. The general case. Let $Z$ be a category which has direct limits $[4$, Def. 9.1]. Then with every covariant functor $\Sigma: \mathcal{U} \rightarrow Z$, i.e. object of the category $Z_{V}[4, \S 7]$ we will associate two covariant functors

$$
\otimes \Sigma: \varsigma \rightarrow Z, \quad H^{v}(\Sigma,): Z \rightarrow \varsigma
$$

where $\otimes \Sigma$ is a left adjoint of $H^{v}(\Sigma, \quad)$ [4, Def. 3.1]. Conversely every pair of covariant functors

$$
S: \AA \rightarrow Z, \quad T: \mathcal{Z} \rightarrow \mathcal{S}
$$

where $S$ is a left adjoint of $T$ may (up to natural equivalences) be obtained in this manner.

Because $\mathrm{Z}$ has direct limits, the embedding functor $E_{d}: \mathrm{Z} \rightarrow \mathrm{Z}_{d}$ has a left adjoint [4, Th. 7.8]. Let $\lim _{d}: Z_{d} \rightarrow Z$ be an arbitrary but fixed such left adjoint and let $\alpha_{d}$ be an arbitrary but fixed natural equivalence $\alpha_{d}: \lim _{d}\left(Z_{d}\right)$ $\dashv E_{d}(\mathrm{Z})$. Let the functor $\otimes_{d}: \mathfrak{N}^{V}, \mathrm{Z}_{V} \rightarrow \mathrm{Z}_{d}$ and the natural transformation

$$
\gamma: H\left(\mathfrak{N}^{V} \otimes_{d} \mathrm{Z}_{V}, E_{d}(\mathrm{Z})\right) \rightarrow H\left(\mathfrak{N}^{v}, H^{v}\left(\mathrm{Z}_{V}, \mathrm{Z}\right)\right)
$$

be as defined in $[4, \S 14]$. Composition of the natural equivalence $\alpha_{d}$ with the functor $\otimes_{d}$ yields a natural equivalence

$$
\alpha_{d} \otimes_{d}: H\left(\lim _{d}\left(\mathfrak{T}^{V} \otimes_{d} \mathrm{Z}_{V}\right), \mathrm{Z}\right) \rightarrow H\left(\mathfrak{T}^{V} \otimes_{d} \mathrm{Z}_{V}, E_{d}(\mathrm{Z})\right) .
$$

Composition of the natural equivalences $\alpha_{d} \otimes_{d}$ and $\gamma$ yields the natural equivalence (see $[4$, Th. 14.1])

$$
\beta: H\left(\lim _{d}\left(\mathfrak{T}^{V} \otimes_{d} \mathrm{Z}_{V}\right), \mathrm{Z}\right) \rightarrow H\left(\mathscr{N}^{V}, \mathrm{H}^{V}\left(\mathrm{Z}_{V}, \mathrm{Z}\right)\right) .
$$


It follows that $\beta$ is completely determined by the choice of $\lim _{d}$ and $\alpha_{d}$; a different choice of $\lim _{d}$ and $\alpha_{d}$ changes $\beta$ by a unique natural equivalence $[4$, Theorem $\left.\left(4.4^{*}\right)\right]$.

Now denote by

$$
\otimes: \mathfrak{T}^{V}, \mathrm{Z}_{V} \rightarrow \mathrm{Z}
$$

the composite functor $\lim _{d} \otimes_{d}: \mathfrak{M}^{V}, \mathrm{Z}_{V} \rightarrow \mathrm{Z}$ and write $S$ instead of $\mathfrak{M}^{V}$. Then $\beta$ is a natural equivalence

$$
\beta: H\left(\mathcal{S} \otimes \mathrm{Z}_{V}, \mathrm{Z}\right) \rightarrow H\left(\mathcal{S}, H^{V}\left(\mathrm{Z}_{V}, \mathrm{Z}\right)\right) .
$$

Hence, given the functor $\lim _{d}: Z_{d} \rightarrow Z$ and the natural equivalence $\alpha_{d}: \lim _{d} \dashv E_{d}$, we may associate with every object $\Sigma \in Z_{V}$

(i) the covariant functor

$$
H^{v}(\Sigma,): \mathrm{Z} \rightarrow \mathrm{s}
$$

which is the right adjoint of

(ii) the covariant functor

$\otimes \Sigma: S \rightarrow Z$

under

(iii) the natural equivalence

$$
\beta_{\Sigma}=\beta(\mathcal{S}, \Sigma, \mathrm{Z}): H(\mathcal{S} \otimes \Sigma, \mathrm{Z}) \rightarrow H\left(\mathcal{S}, H^{v}(\Sigma, \mathrm{Z})\right),
$$

(iv) the natural transformation induced by $\beta_{\Sigma}$

$$
\kappa_{\Sigma}: E(s) \rightarrow H^{v}(\Sigma, s \otimes \Sigma)
$$

satisfying the relation

$$
\beta_{\Sigma} f=H^{v}(\Sigma, f) \circ \kappa_{\Sigma} K
$$

for every object $K \in \mathcal{S}$ and $Z \in Z$ and every map $f: K \otimes \Sigma \rightarrow Z \in \mathcal{Z}$ [4, Lemma 6.2 ], and

(v) the natural transformation induced by $\beta_{\Sigma}^{-1}$

$$
\mu_{\Sigma}: H^{v}(\Sigma, \mathrm{Z}) \otimes \Sigma \rightarrow E(\mathrm{Z})
$$

satisfying the relation

$$
\beta_{\Sigma}^{-1} g=\mu_{\Sigma} Z \circ g \otimes \Sigma
$$

for every object $K \in \mathcal{S}$ and $Z \in \mathcal{Z}$ and every map $g: K \rightarrow H^{v}(\Sigma, Z) \in \mathcal{S}[4$, Lemma $6.2^{*}$ ].

When no confusion can arise the subscript $\Sigma$ will be omitted in $\beta_{\Sigma}, \kappa_{\Sigma}$ and $\mu_{\Sigma}$.

By a suitable choice of the category $Z$ and the object $\Sigma \in Z_{V}$ the above functors and natural transformations reduce to well known ones.

That every pair of covariant functors $S: \mathcal{S} \rightarrow \mathrm{Z}$ and $T: \mathrm{Z} \rightarrow \mathcal{S}$ where $S$ is a 
left adjoint of $T$ may (up to natural equivalences) be obtained in the above manner can be seen as follows. Consider the identity functor $E: \vartheta \rightarrow U$ as an object of the category $v_{V}$. Then the lifted functor $H^{v}: V_{V}, \vartheta \rightarrow \mathscr{N}^{V}$ induces a functor $H^{V}(E, \quad): \mathcal{U} \rightarrow \mathcal{S}\left(\mathfrak{T M}^{V}=S\right)$. Let $\Sigma: \mathcal{U} \rightarrow \mathrm{Z}$ be the composite functor

$$
v \stackrel{H^{v}(E,)}{\longrightarrow} \mathrm{s} \stackrel{S}{\longrightarrow} \mathrm{Z} .
$$

Then it is readily verified, using the fact that $S$ is a left adjoint of $T$, that there exists a natural equivalence $\tau: H^{v}(\Sigma, \quad) \rightarrow T$ and hence [4, Th. (3.2*)] implies the existence of a natural equivalence $\sigma: S \rightarrow \otimes \Sigma$.

REMARK. The results of this section also hold if $V$ is replaced by any other proper category $\mathcal{u}$ and $\delta$ by $\mathfrak{N}^{U}$.

4. Topological spaces. Let $a$ be the category of topological spaces and and continuous maps

Proposition (4.1). The category a has direct limits.

Proof. Let $\mathcal{u}$ be a proper category and let $K: \mathcal{u} \rightarrow Q$ be a covariant functor $\cdot$ Denote by $B$ the set of all pairs $(U, x)$ where $U \in \mathcal{U}$ is an object and $x \in K U$ a point. Define a relation $(U, x) \sim\left(U^{\prime}, x^{\prime}\right)$ if there exists a map $u: U \rightarrow U^{\prime} \in \mathcal{u}$ such that $(K u) x=x^{\prime}$. This relation $\sim$ induces an equivalence relation on $B$. Let $A$ be the set of all equivalence classes and for every object $U \in \mathcal{U}$ let

$$
k_{U}: K U \rightarrow A
$$

be the function which assigns to a point $x \in K U$ the equivalence class of $(U, x)$. Introduce a topology in $A$ by defining a subset $M \subset A$ open if for every object $U \in U$

$$
k_{U}^{-1}\left(M \cap k_{U}(K U)\right)
$$

is open in $K U$. It is now easily verified that

$$
A=\lim _{k} K
$$

where the map $k: K \rightarrow E_{U} A$ is given by $k U=k_{U}$ for every object $U \in \mathcal{u}$. This completes the proof.

It should be noted (see [4, Remark 7.9]) that in the proof of Proposition 4.1 not merely the existence of a direct limit is established but that a procedure is given by which simultaneously for all pairs $(\mathcal{u}, K)$, where $\mathcal{u}$ is a proper category and $K: น \rightarrow Q$ a covariant functor, an object $A$ and a map $k: K \rightarrow E_{U} A$ can be found such that $A=\lim _{k} K$. Let the functor

$$
\lim _{d}=a_{d} \rightarrow Q
$$

and the natural equivalence

$$
\alpha_{d}: H\left(\lim _{d}\left(Q_{d}\right), a\right) \rightarrow H\left(Q_{d}, E_{d}(Q)\right)
$$


be the unique ones induced by the above procedure of assigning to every pair $(\mathcal{U}, K)$ an object $A$ and a map $k: K \rightarrow E_{U} A$ (see [4, Theorem 9.5]).

Define an object $\Sigma \in Q_{V}$ as follows. For each integer $n \geqq 0, \Sigma[n]$ is an euclidean $n$-simplex with ordered vertices $A_{0}, \cdots, A_{n}$; for each map $\alpha:[m]$ $\rightarrow[n], \Sigma \alpha: \Sigma[m] \rightarrow \Sigma[n]$ is the simplicial map defined by $(\Sigma \alpha) A_{i}=A_{\alpha(i)}$ for all $0 \leqq i \leqq m$.

The following then can readily be verified by comparison with the usual definitions.

Proposition (4.2). For every topological space $X$

$$
H^{v}(\Sigma, X)
$$

is its simplicial singular complex (see [2]).

Proposition (4.3). For every c.s.s. complex $K$

$K \otimes \Sigma$

is its geometrical realization (by a $C W$-complex of which the $n$-cells are in oneto-one correspondence with the nondegenerate simplices of $K$; (see [6]).

The existence of the (natural) equivalence

$$
\beta: H(K \otimes \Sigma, X) \rightarrow H\left(K, H^{v}(\Sigma, X)\right)
$$

expresses the fact that for every object $K \in \mathcal{S}$ and $X \in Q$ there exists a one-toone correspondence between the continuous maps $K \otimes \Sigma \rightarrow X$ and the c.s.s. maps $K \rightarrow H^{v}(\Sigma, X)$.

Proposition (4.4). For every c.s.s. complex $K$

$$
\kappa K: K \rightarrow H^{v}(\Sigma, K \otimes \Sigma)
$$

is the (natural) embedding of $K$ into the simplicial singular complex of its geometrical realization (see [6]).

Proposition (4.5). For every topological space $X$

$$
\mu X: H^{v}(\Sigma, X) \otimes \Sigma \rightarrow X
$$

is the (natural) map of the geometrical realization of the simplicial singular complex of $X$ onto $X$ (see [6]).

For a locally compact space $Y \in Q$ define another object $\Sigma_{Y} \in Q_{V}$ by

$$
\Sigma_{Y}[n]=\Sigma[n] \times Y ; \quad\left(\Sigma_{Y}\right) \alpha=\Sigma \alpha \times i_{Y}
$$

where $X$ denotes the cartesian product. As for every two topological spaces $X$ and $Z$ there exists a (natural) equivalence

$$
H(Z \times Y, X) \approx H\left(Z, X^{Y}\right)
$$


where $X^{Y}$ denotes the function space with the compact open topology, it follows immediately that

Proposition (4.6). For every topological space $X$

$$
H^{v}\left(\Sigma_{Y}, X\right) \approx H^{v}\left(\Sigma, X^{Y}\right) .
$$

Similarly because the operation of "taking the direct limit" commutes with "taking the cartesian product with $Y$ " it follows that

Proposition (4.7). For every c.s.s. complex $K$

$$
K \otimes \Sigma_{Y} \approx(K \otimes \Sigma) \times Y .
$$

\section{C.s.s. complexes.}

Proposition (5.1). The category $S$ has direct limits.

Proof. This follows immediately from [4, Corollary 12.2] and the following proposition.

Proposition (5.2). The category $\mathfrak{T}$ has direct limits.

Proof. Omit all mention of topology from the proof of Proposition 4.1.

Define an object $\Delta \in \mathcal{S}_{V}$ as follows. Consider the identity functor $E: \mathcal{U} \rightarrow \mathcal{U}$ as an object of the category $\vartheta_{V}$ and define, using the lifted functor $H^{v}: V_{V}$, $v \rightarrow \mathbb{N}^{V}$

$$
\Delta[n]=H^{v}(E,[n]) ; \quad \Delta \alpha=H^{v}(E, \alpha) .
$$

Thus $\Delta[n]$ is the standard $n$-simplex (this is $K[n]$ in the notation of EilenbergZilber, see $[2 ; 3])$.

The functors associated with the object $\Delta \in \delta_{V}$ are, up to a natural equivalence, the identity. For every $n$-simplex $\sigma$ of a c.s.s. complex $K$ let $\phi_{\sigma}: \Delta[n] \rightarrow K$ denote the unique map such that $\phi_{\sigma} \alpha=\sigma \alpha$ for all $\alpha \in \Delta[n]$. It then follows easily from the uniqueness of the map $\phi_{o}$ that

Proposition (5.3). The function

$$
\phi K: K \rightarrow H^{v}(\Delta, K)
$$

given by

$$
(\phi K) \sigma=\phi_{\sigma} \quad \sigma \in K
$$

is an isomorphism. This isomorphism is natural.

Similarly it can be shown:

Proposition (5.4). The map

$$
\beta^{-1}(\phi K): K \otimes \Delta \rightarrow K
$$

is a (natural) isomorphism. 
For a c.s.s. complex $L$ define another object $\Delta_{L} \in S_{V}$ by

$$
\Delta_{L}[n]=\Delta[n] \times L, \quad\left(\Delta_{L}\right) \alpha=\Delta \alpha \times i_{L}
$$

where $X$ denotes the cartesian product. Then Proposition 5.4 together with the fact that "taking the direct limit" commutes with "taking the cartesian product with $L$ " yields

Proposition (5.5). For every c.s.s. complex $K$

$$
K \otimes \Delta_{L} \approx K \times L .
$$

For $L, M \in \mathcal{S}$ define the function complex $M^{L}$ by

$$
M^{L}=H^{v}\left(\Delta_{L}, M\right) \text {. }
$$

"Taking the cartesian product" is thus a left adjoint of "taking the function complex." In fact we have

Proposition (5.6). Let $K, L$ and $M \in S$. Then there exists a (natural) equivalence

$$
M^{K \times L} \approx\left(M^{L}\right)^{K} .
$$

The functors associated with still another choice of an object of $S_{V}$ will be considered in [5].

6. Chain complexes. Let $\partial g$ be the category of chain complexes and chain maps.

Proposition (6.1). The category $\partial \mathcal{G}$ has direct limits.

In order to prove this we need

Proposition (6.2). The category 9 of abelian groups has direct limits.

Proof of Proposition (6.2). Let $\mathfrak{u}$ be a proper category and let $K: \mathfrak{u} \rightarrow \mathcal{G}$ be a covariant functor. Let $A$ be the abelian group generated by the pairs $(U, x)$ where $U \in \mathcal{U}$ is an object and $x \in K U$ an element, with the following relations: for every object $U \in \mathcal{u}$ and every two elements $x, y \in K U$

$$
(U, x)+(U, y)=(U, x+y)
$$

and for every map $u: U \rightarrow U^{\prime} \in \mathcal{u}$ and every element $x \in K U$

$$
(U, x)=\left(U^{\prime},(K u) x\right) .
$$

It follows from the first kind of relations that for every object $U \in \mathcal{U}$ the function $k_{U}: K U \rightarrow A$ given by $k_{U} x=(U, x)$ is a homomorphism. Straightforward computation now yields that

$$
A=\lim _{k} K
$$

where the map $k: K \rightarrow E_{U} A$ is given by $k U=k_{U}$ for every object $U \in \mathcal{u}$. 
Proof of Proposition (6.1). Let $\mathrm{Z}$ be the category of which the objects are the integers and which contains one map $m \rightarrow n$ for each pair of integers $(m, n)$ with $m \leqq n$. It then follows from Proposition (6.2) and [4], Corollary (12.2) that the category $\mathcal{G}^{z}$ has direct limits. Clearly $\partial \mathcal{g}$ may be considered as a full subcategory of $g^{Z}$ and $\partial \mathcal{G}_{d}$ as a full subcategory of $\mathcal{G}_{d}^{Z}$. It is easily seen that a functor $\lim _{d}: \mathcal{G}_{d}^{Z} \rightarrow \mathcal{G}^{Z}$ maps any object of $\partial \mathcal{G}_{d}$ in to $\partial \mathcal{G}$. Hence the category $\partial \mathcal{G}$ has direct limits.

Define an object $\Gamma \in \partial \mathcal{G}_{v}$ by

$$
\Gamma[n]=C_{N} \Delta[n], \quad \Gamma \alpha=C_{N} \Delta \alpha
$$

where $C_{N}: \varsigma \rightarrow \partial \mathcal{G}$ is the normalized chain functor (see [2]). Of the two adjoint functors associated with $\Gamma$ one, $\otimes \Gamma$, is, up to a natural equivalence, the normalized chain functor $C_{N}$; the other functor $H^{v}(\Gamma, \quad)$, will be investigated in the remainder of this paper.

Proposition (6.3). There exists a natural equivalence

$$
c: S \otimes \Gamma \rightarrow C_{N}(S) .
$$

Proof. Let $K \in \delta$ be an object. Define a map $f: K \otimes_{d} \Gamma \rightarrow E_{d} C_{N} K$ by $f([n], \sigma)$ $=C_{N} \phi_{\sigma}$ for every $n$-simplex $\sigma \in K$, where $\phi_{\sigma}: \Delta[n] \rightarrow K$ is the unique map such that $\phi_{\sigma} \alpha=\sigma \alpha$ for all $\alpha \in \Delta[n]$. Let $c: K \otimes \Gamma \rightarrow C_{N} K$ be the unique map such that $f=E_{d} c \circ \lambda_{d}\left(K \otimes_{d} \Gamma\right)$, where $\lambda_{d}: E\left(\partial \mathcal{G}_{d}\right) \rightarrow E_{d} \lim _{d}\left(\partial \mathcal{G}_{d}\right)$ is the natural transformation induced by the natural equivalence $\alpha_{d}: \lim _{d}\left(\partial \mathcal{G}_{d}\right) \dashv E_{d}(\partial \mathcal{G})$. Straightforward computation then yields that $c$ is an isomorphism. Naturality now follows easily.

7. Some definitions and lemmas. We shall now state several definitions and lemmas which will be needed in the sequel. For proofs, see [7].

For every pair of integers $(k, n)$ with $0 \leqq k \leqq n$ let $\epsilon^{k}:[n-1] \rightarrow[n]$ be the monotone function given by

$$
\begin{array}{rlrl}
\epsilon^{k}(i) & =i & i<k \\
\epsilon^{k}(i) & =i+1 & i \geqq k
\end{array}
$$

and let $\eta^{k}:[n] \rightarrow[n-1]$ be given by

$$
\begin{array}{rlrl}
\eta^{k}(i) & =i & i \leqq k \\
\eta^{k}(i) & =i-1 & i>k .
\end{array}
$$

Definition (7.1). A c.s.s. complex $K$ is said to satisfy the extension condition if for every pair of integers $(k, n)$ with $0 \leqq k \leqq n$ and for every $n(n-1)$ simplices $\sigma_{0}, \cdots, \sigma_{k-1}, \sigma_{k+1}, \cdots, \sigma_{n} \in K$ such that $\sigma_{i} \epsilon^{i-1}=\sigma_{j} \epsilon^{i}$ for $i<j$ and $i \neq k \neq j$, there exists an $n$-simplex $\sigma \in K$ such that $\sigma \epsilon^{i}=\sigma_{i}$ for $i=0, \cdots$, $\hat{k}, \cdots, n$.

Denote by $\delta_{E}$ the full subcategory of $\delta$ generated by the c.s.s. complexes which satisfy the extension condition. 
Definition (7.2). Two $n$-simplices $\sigma$ and $\tau$ of a c.s.s. complex $K$ are called homotopic (notation $\sigma \sim \tau$ ) if

(i) their faces coincide, i.e. $\sigma \epsilon^{i}=\sigma \epsilon^{i}$ for all $i$,

(ii) there exists an $(n+1)$-simplex $\rho \in K$ such that

$$
\begin{aligned}
\rho \epsilon^{0} & =\sigma, \\
\rho \epsilon^{1} & =\tau, \\
\rho \epsilon^{i+1} & =\sigma \epsilon^{i} \eta^{0}=\tau \epsilon^{i} \eta^{0}
\end{aligned}
$$$$
0<i \leqq n .
$$

Lemma (7.3). If $K \in S_{E}$, then $\sim$ is an equivalence relation on the simplices of $K$.

Definition (7.4). Let $K \in S_{E}$ and let $\phi \in K$ be a 0 -simplex. For every integer $n \geqq 0$ a group $\pi_{n}(K ; \phi)$, the nth homotopy group of $K$ rel. $\phi$ will be defined as follows. Consider the collection $\Sigma$ of the $n$-simplices $\sigma \in K$ such that

$$
\sigma \epsilon^{i}=\phi \eta^{0} \cdots \eta^{n-2} \quad 0 \leqq i \leqq n .
$$

The equivalence relation $\sim$ divides $\Sigma$ into classes. These classes $a, b$, etc. will be the elements of $\pi_{n}(K ; \phi)$. Now let $\sigma \in a$ and $\tau \in b$ be arbitrary representatives of the classes $a$ and $b$. Because $K \in \delta_{E}$ there exists an $(n+1)$ simplex $\rho \in K$ such that

$$
\begin{aligned}
\rho \epsilon^{0} & =\sigma, \\
\rho \epsilon^{2} & =\tau, \\
\rho \epsilon^{i} & =\phi \eta^{0} \cdots \eta^{n-1}
\end{aligned}
$$$$
2<i \leqq n+1 \text {. }
$$

The sum $a+b$ of the classes $a$ and $b$ then is defined as the class of the $n$-simplex $\rho \epsilon^{1}$. It can be shown that $\pi_{n}(K ; \phi)$ so defined is a group and is independent of the different choices made in its definition.

Definition (7.5). Let $\mathfrak{F}$ be the category of groups and homomorphisms. The objects of the category $\mathrm{JC}^{\mathrm{V}}$ will be called c.s.s. groups and the maps of $\mathrm{JC}^{V}$ c.s.s. homomorphisms. A c.s.s. group $G$ thus is a c.s.s. complex such that

(i) $G[n]$ is a group for each integer $n \geqq 0$,

(ii) $G \alpha: G[n] \rightarrow G[m]$ is a homomorphism for every map $\alpha:[m] \rightarrow[n] \in v$. The objects of the category $\mathcal{G}^{V}$ ( $\mathcal{G}$ is the category of abelian groups) will be called c.s.s. abelian groups.

The following results are due to J. C. Moore. (see [7]).

LEMMA (7.6). Every c.s.s. group satisfies the extension condition.

Let $G$ be a c.s.s. group. For each integer $n \geqq 0$ define a subgroup $\widetilde{G}_{n} \subset G[n]$ by

$$
\widetilde{G}_{n}=\bigcap_{i=1}^{n} \text { kernel } G \epsilon^{i}
$$

Then $\sigma \in \widetilde{G}_{n+1}$ implies $\sigma \epsilon^{0} \in \widetilde{G}_{n}$. Define a homomorphism $\widetilde{\partial}_{n+1}: \widetilde{G}_{n+1} \rightarrow \widetilde{G}_{n}$ by 
$\tilde{\partial}_{n+1} \sigma=\sigma \epsilon^{0}$, i.e. $\tilde{\partial}_{n+1}=G \epsilon^{0} \mid \tilde{G}_{n+1}$. For each integer $n<0$ let $G_{n}=1$ and let $\tilde{\partial}_{n+1}: \widetilde{G}_{n+1} \rightarrow \widetilde{G}_{n}$ be the trivial homomorphism. It may be shown that for all $n$ image $\tilde{\partial}_{n+1}$ is a normal subgroup of kernel $\partial_{n}$, i.e. $\widetilde{G}=\left\{\widetilde{G}_{n}, \tilde{\partial}_{n}\right\}$ is a (not necessary abelian) chain complex. Its homology groups are

$$
H_{n}(G)=\text { kernel } \tilde{\partial}_{n} / \text { image } \tilde{\partial}_{n+1} \text {. }
$$

Let $G$ be a c.s.s. group and let $e \in G[0]$ be the identity. The homotopy group $\pi_{n}(G ; e)$ will of ten be denoted by $\pi_{n}(G)$. The group structure of $G[0]$ induces a group structure in $\pi_{0}(G)=\pi_{0}(G ; e)$, the set of the components of $G$ and we have

Lemma (7.7). $\pi_{n}(G)=H_{n}(\tilde{G})$ for each integer $n \geqq 0$.

Let $G$ be a c.s.s. group and let $\phi \in G[0]$. For each $n$-simplex $\sigma \in G$ denote by $\phi \# \sigma$ the $n$-simplex

$$
\phi_{\sharp \sigma}=\sigma\left(\phi^{-1} \eta^{0} \cdots \eta^{n-1}\right) .
$$

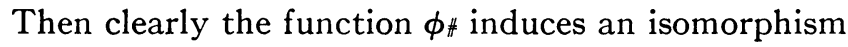

$$
\phi_{*}: \pi_{n}(G ; \phi) \rightarrow \pi_{n}(G) .
$$

8. The functor $H^{v}(\Gamma$,$) . For every object A$ in $\partial \mathcal{G}$, the category of (abelian) chain complexes, the c.s.s. complex $H^{v}(\Gamma, A)$ may be converted into a c.s.s. abelian group as follows. Let $\sigma, \tau: \Gamma[n] \rightarrow A$ be two $n$-simplices of $H^{v}(\Gamma, A)$. Then the sum $\sigma+\tau: \Gamma[n] \rightarrow A$ is defined by

$$
(\sigma+\tau) \gamma=\sigma \gamma+\tau \gamma \quad \gamma \in \Gamma[n] .
$$

For every chain map $f: A \rightarrow B$ the c.s.s. map $H^{v}(\Gamma, f): H^{v}(\Gamma, A) \rightarrow H^{v}(\Gamma, B)$ then becomes a c.s.s. homomorphism. Hence $H^{v}(\Gamma, \quad)$ may be regarded as a functor

$$
H^{V}(\Gamma,): \partial \mathcal{G} \rightarrow \mathcal{S}^{V} .
$$

Let $\partial \mathcal{G}^{0}$ be the full subcategory of $\partial \mathcal{G}$ generated by the chain complexes which are 0 in dimension $<0$, i.e. $A \in \partial \mathcal{G}^{0}$ if and only if $A_{i}=0$ for $i<0$. Let

$$
M: \mathcal{S}^{V} \rightarrow \partial \mathcal{G}^{0}
$$

be the functor which assigns to every c.s.s. abelian group $G$ the chain complex $M G=\widetilde{G}$ and to every c.s.s. homomorphism $f: G \rightarrow H$ the chain map $M f: \widetilde{G} \rightarrow \widetilde{H}$ given by $(M f) \sigma=f \sigma$ for $\sigma \in \tilde{G}$.

Roughly speaking, the functor $H^{v}(\Gamma, \quad)$ sets up a one-to-one correspondence between the objects and maps of $\partial \mathcal{G}^{0}$ and those of $\mathcal{G}^{V}$. An exact formulation of both halves of this statement is given in the following two theorems, in which $E$ denotes the identity functor.

THEOREM (8.1). There exists a natural equivalence

$$
a: M H^{v}\left(\Gamma, \partial \mathcal{G}^{0}\right) \rightarrow E\left(\partial \mathcal{G}^{0}\right) .
$$


THEOREM (8.2). There exists a natural equivalence

$$
b: E\left(\mathcal{G}^{V}\right) \rightarrow H^{v}\left(\Gamma, M\left(\mathcal{S}^{V}\right)\right) .
$$

Let $A \in \partial \mathcal{G}$ and let $A^{\circ} \subset A$ be the subcomplex given by

$$
\begin{array}{ll}
A_{n}^{0}=A_{n} & n>0, \\
A_{0}^{0}=\partial_{0}^{-1}(0), & \\
A_{n}^{0}=0 & n<0 .
\end{array}
$$

It is readily verified that

$$
H^{v}(\Gamma, A)=H^{V}\left(\Gamma, A^{0}\right)
$$

and that the inclusion map $j: A^{0} \rightarrow A$ induces isomorphisms

$$
j_{*}: H_{n}\left(A^{0}\right) \rightarrow H_{n}(A)
$$$$
n \geqq 0 .
$$

Application of Theorem 8.1 and Lemma 7.7 now yields

Corollary (8.3). Let $A \in \partial g$. Then for every integer $n \geqq 0$

$$
j_{*} a_{*}: \pi_{n}\left(H^{V}(\Gamma, A)\right) \approx H_{n}(A)
$$

i.e. the nth homotopy group of the c.s.s. group $H^{v}(\Gamma, A)$ is isomorphic with the nth homology group of the chain complex $A$.

Proof of Theorem (8.1). For each nondegenerate simplex $\alpha \in \Delta[n]$ let $C_{N} \alpha$ be the corresponding generator of $\Gamma[n]$. Let $A \in \partial \mathcal{G}^{\circ}$ be an object and let $G=H^{v}(\Gamma, A)$. For each simplex $\sigma: \Gamma[n] \rightarrow A \in \widetilde{G}_{n}$ define an element $a \sigma \in A_{n}$ by

$$
a \sigma=\sigma\left(C_{n} \epsilon_{n}\right),
$$

where $\epsilon_{n}:[n] \rightarrow[n]$ is the identity map, i.e. the only nondegenerate $n$-simplex of $\Delta[n]$. As the addition in $G$ was induced by that of $A$ it follows that the function $a: \widetilde{G}_{n} \rightarrow A_{n}$ is a homomorphism for each $n$.

It follows from the definition of $\widetilde{G}_{n}$ that a simplex $\sigma: \Gamma[n] \rightarrow A$ is in $\widetilde{G}_{n}$ if and only if $\sigma \epsilon^{i}: \Gamma[n-1] \rightarrow A$ is the zero map for $i \neq 0$, i.e. $\sigma$ maps all generators of $\Gamma[n]$, with the possible exception of $C_{N} \epsilon_{n}$ and $C_{N} \epsilon^{0}$, into zero. Consequently

$$
\begin{aligned}
\partial_{n}(a \sigma) & =\partial_{n}\left(\sigma\left(C_{N} \epsilon_{n}\right)\right)=\sum(-1)^{i}\left(\sigma\left(C_{N} \epsilon^{i}\right)\right) \\
& =\sigma\left(C_{N} \epsilon^{0}\right)=\left(\sigma \epsilon^{0}\right)\left(C_{N} \epsilon_{n-1}\right) \\
& =\left(\tilde{\partial}_{n} \sigma\right)\left(C_{N} \epsilon_{n-1}\right)=a\left(\tilde{\partial}_{n} \sigma\right),
\end{aligned}
$$

i.e. the function $a: \widetilde{G} \rightarrow A$ is a chain map. It also follows that $\sigma$ is completely determined by $\sigma\left(C_{N} \epsilon_{n}\right) \in A_{n}$. Hence $a: \widetilde{G} \rightarrow A$ is an isomorphism. Naturality is easily verified.

The proof of Theorem (8.2) will be given in $\$ 11$. 
Let $X$ be a topological space. The homotopy groups of $X$ are by definition those of its simplicial singular complex $K=H^{v}(\Sigma, X)$ (see $\S 4$ ), and the singular homology groups of $X$ are the homology groups of the chain complex $C_{N} K$. Let $\phi$ be a 0 -simplex of $K$, let

$$
h_{*}: \pi_{n}(K ; \phi) \rightarrow H_{n}\left(C_{N} K\right)
$$

be the Hurewicz homomorphism and let

$$
\kappa: E(\$) \rightarrow H^{v}(\Gamma, s \otimes \Gamma)
$$

be the natural transformation induced by the natural equivalence $\beta: s \otimes \Gamma$ $\dashv H^{v}(\Gamma, \partial \mathcal{G})$. Then it can be shown that

Proposition (8.4). Commutativity holds in the diagram

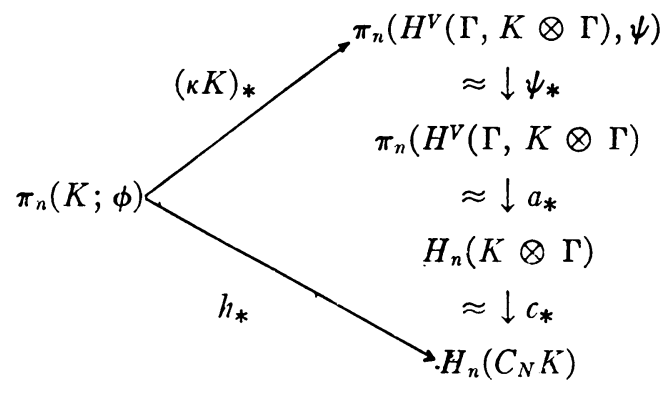

where $\psi=(\kappa K) \phi$, i.e. the map $\kappa K: K \rightarrow H^{v}(\Gamma, K \otimes \Gamma$ ) induces (up to an equivalence) the Hurewicz homomorphisms.

9. The $K(\pi, n)$. Let $\pi$ be an abelian group and let $n$ be an integer $\geqq 0$. Denote by $(\pi, n)$ the chain complex with $\pi$ in dimension $n$ and 0 in the others.

Proposition (9.1). $H^{v}(\Gamma,(\pi, n))=K(\pi, n)$, the Eilenberg-MacLane complex of $\pi$ on level $n$ (see [1]).

Proof. A $q$-simplex of $K(\pi, n)$ is an element of $Z^{n}(\Delta[q], \pi)$, i.e. a chain map $\Gamma[q] \rightarrow(\pi, n)$ and hence a $q$-simplex of $H^{V}(\Gamma,(\pi, n))$ and conversely. It is readily verified that this one-to-one correspondence commutes with all operators $\alpha$.

Combination of Proposition (9.1) with Lemma (7.7) yields

CoRollary (9.2). $\pi_{n}(K(\pi, n))=\pi ; \pi_{i}(K(\pi, n))=0$ for $i \neq n$.

Let $K$ be a c.s.s. complex. Then combination of the equivalence

$$
\beta: H(K \otimes \Gamma,(\pi, n)) \rightarrow H\left(K, H^{v}(\Gamma,(\pi, n))\right.
$$

with the equivalence $c: K \otimes \Gamma \rightarrow C_{N} K$ of Proposition 6.3 yield an equivalence

$$
\beta^{\prime}: H\left(C_{N} K,(\pi, n)\right) \rightarrow H(K, K(\pi, n))
$$

which expresses the well known fact that the elements of $Z^{n}(K ; \pi)$, i.e. the 
chain maps $C_{N} K \rightarrow(\pi, n)$ are in one-to-one correspondence with the c.s.s. maps $K \rightarrow K(\pi, n)$.

10. Two lemmas. We shall now give two lemmas which will be needed in the proof of Theorem (8.2).

Let $G$ be a c.s.s. abelian group and let $\sigma_{1}, \cdots, \sigma_{n} \in G[n-1]$ be such that $\sigma_{i} \epsilon^{j-1}=\sigma_{j} \epsilon^{i}$ for $0<i<j$. Let

$$
\begin{aligned}
& \phi_{n}=\sigma_{n} \eta^{n-1}, \\
& \phi_{k}=\sigma_{k} \eta^{k-1}-\phi_{k+1} \epsilon^{k} \eta^{k-1}+\phi_{k+1}
\end{aligned}
$$$$
0<k<n
$$

and define an $n$-simplex $\phi\left(\sigma_{1}, \cdots, \sigma_{n}\right) \in G[n]$ by

$$
\phi\left(\sigma_{1}, \cdots, \sigma_{n}\right)=\phi_{1} .
$$

Lemma (10.1). $\phi\left(\sigma_{1}, \cdots, \sigma_{n}\right) \epsilon^{i}=\sigma_{i}$ for $i \neq 0$.

Lemma (10.2). Let $\tau \in G[n-1]$. Then

$$
\phi\left(\tau \eta^{i} \epsilon^{1}, \cdots, \tau \eta^{i} \epsilon^{n}\right)=\tau \eta^{i} \quad \text { for } 0 \leqq i \leqq n-1 .
$$

Proof of Lemma (10.1). Clearly

$$
\begin{aligned}
& \phi_{n} \epsilon^{n}=\sigma_{n} \eta^{n-1} \epsilon^{n}=\sigma_{n}, \\
& \phi_{k} \epsilon^{k}=\sigma_{k} \eta^{k-1} \epsilon^{k}-\phi_{k+1} \epsilon^{k} \eta^{k-1} \epsilon^{k}+\phi_{k+1} \epsilon^{k}=\sigma_{k} .
\end{aligned}
$$

If $1<k \leqq i$, then

$$
\begin{aligned}
\phi_{k-1} \epsilon^{i} & =\sigma_{k-1} \eta^{k-2} \epsilon^{i}-\phi_{k} \epsilon^{k-1} \eta^{k-2} \epsilon^{i}+\phi_{k} \epsilon^{i} \\
& =\sigma_{k-1} \epsilon^{i-1} \eta^{k-2}-\phi_{k} \epsilon^{i} \epsilon^{k-1} \eta^{k-2}+\phi_{k} \epsilon^{i} \\
& =\sigma_{i} \epsilon^{k-1} \eta^{k-2}-\sigma_{i} \epsilon^{k-1} \eta^{k-2}+\sigma_{i}=\sigma_{i} .
\end{aligned}
$$

Hence $\phi\left(\sigma_{1}, \cdots, \sigma_{n}\right) \epsilon^{i}=\phi_{1} \epsilon^{i}=\sigma_{i}$ for $i \neq 0$.

Proof of Lemma (10.2). If $\phi_{k+1}=\tau \eta^{i}$ for some $k$, then

$$
\begin{aligned}
\phi_{k} & =\tau \eta^{i} \epsilon^{k} \eta^{k-1}-\phi_{k+1} \epsilon^{k} \eta^{k-1}+\phi_{k+1} \\
& =\tau \eta^{i} \epsilon^{k} \eta^{k-1}-\tau \eta^{i} \epsilon^{k} \eta^{k-1}+\tau \eta^{i}=\tau \eta^{i} .
\end{aligned}
$$

Hence it suffices to show that $\phi_{i+1}=\tau \eta^{i}$ for all $i$. For $i=n-1$

$$
\phi_{n}=\tau \eta^{n-1} \epsilon^{n} \eta^{n-1}=\tau \eta^{n-1} .
$$

Let

$$
\begin{aligned}
\psi_{n-1} & =\tau \epsilon^{n-1} \eta^{n-2}, \\
\psi_{k} & =\tau \epsilon^{k} \eta^{k-1}-\psi_{k+1} \epsilon^{k} \eta^{k-1}+\psi_{k+1} \quad \text { for } 0<k<n-1 .
\end{aligned}
$$

Then for $i<n-1$

$$
\phi_{n}=\tau \eta^{i} \epsilon^{n} \eta^{n-1}=\tau \epsilon^{n-1} \eta^{n-2} \eta^{i}=\psi_{n-1} \eta^{i}
$$

and if $\phi_{k}=\psi_{k-1} \eta^{i}$ for $i+2<k \leqq n$, then 


$$
\begin{aligned}
\phi_{k-1} & =\tau \eta^{i} \epsilon^{k-1} \eta^{k-2}-\phi_{k} \epsilon^{k-1} \eta^{k-2}+\phi_{k} \\
& =\left(\tau \epsilon^{k-2} \eta^{k-3}-\psi_{k-1} \epsilon^{k-2} \eta^{k-3}+\psi_{k-1}\right) \eta^{i}=\psi_{k-2} \eta^{i} .
\end{aligned}
$$

Hence

$$
\begin{aligned}
\phi_{i+1} & =\tau \eta^{i} \epsilon^{i+1} \eta^{i}-\phi_{i+2} \epsilon^{i+1} \eta^{i}+\phi_{i+2} \\
& =\tau \eta^{i}-\psi_{i+1} \eta^{i} \epsilon^{i+1} \eta^{i}+\psi_{i+1} \eta^{i}=\tau \eta^{i} .
\end{aligned}
$$

REMARK. In the proofs of the Lemmas (10.1) and (10.2) no use was made of the fact that $G$ is abelian. Hence both lemmas also hold in the nonabelian case.

11. Proof of Theorem (8.2). Let $G$ be a c.s.s. abelian group and let $H=H^{v}(\Gamma, \tilde{G})$. For each integer $n \geqq 0$ define a function $b: G[n] \rightarrow H[n]$ by

$$
\begin{aligned}
b \sigma & =a^{-1} \sigma & \operatorname{dim} \sigma=0 ; \\
b \sigma & =a^{-1}\left(\sigma-\phi\left(\sigma \epsilon^{1}, \cdots, \sigma \epsilon^{n}\right)\right)+\phi\left(b\left(\sigma \epsilon^{1}\right), \cdots, b\left(\sigma \epsilon^{n}\right)\right) & \operatorname{dim} \sigma>0 .
\end{aligned}
$$

Clearly the function $b: G[0] \rightarrow H[0]$ is an isomorphism because $a$ is so. Now suppose it has already been proved that the functions $b: G[i] \rightarrow H[i]$ are isomorphisms for $i<n$ and commute with all face and degeneracy operators in dimension $<n$, then it must be shown that $b=G[n] \rightarrow H[n]$ is an isomorphism and that $b$ commutes with all face and degeneracy operators in dimension $\leqq n$.

That $b: G[n] \rightarrow H[n]$ is a homomorphism follows from the induction hypothesis and the fact that the function $\phi$ only involves face and degeneracy operators and therefore is additive.

We now first show that $b$ commutes with all face operators in dimension $\leqq n$. It follows from Lemma (10.1) that for $i \neq 0$ :

$$
\begin{aligned}
(b \sigma) \epsilon^{i} & =a^{-1}\left(\sigma-\phi\left(\sigma \epsilon^{1}, \cdots, \sigma \epsilon^{n}\right)\right) \epsilon^{i}+\phi\left(b\left(\sigma \epsilon^{1}\right), \cdots, b\left(\sigma \epsilon^{n}\right)\right) \epsilon^{i} \\
& =a^{-1}\left(\sigma \epsilon^{i}-\sigma \epsilon^{i}\right)+b\left(\sigma \epsilon^{i}\right)=b\left(\sigma \epsilon^{i}\right) .
\end{aligned}
$$

Let $\phi_{1}=\phi\left(\sigma \epsilon^{1}, \cdots, \sigma \epsilon^{n}\right)$. Then it follows from the definition of $b$ that

$$
\left(b \phi_{1}\right) \epsilon^{0}=\phi\left(b\left(\sigma \epsilon^{1}\right), \cdots, b\left(\sigma \epsilon^{n}\right)\right) \epsilon^{0} .
$$

Thus $\left(b \phi_{1}\right) \epsilon^{0}$ is obtained from $\sigma \epsilon^{1}, \cdots, \sigma \epsilon^{n}$ by application of the isomorphism $b: G[n-1] \rightarrow H[n-1]$ and operators $\epsilon^{i}$ and $\eta^{i}$. These operators can always be rearranged in such a manner that first all operators $\epsilon^{i}$ are applied and then all $\eta^{j}$. As in this way only simplices in dimension $<n$ are involved, these operators can now be commuted with the isomorphism $b$ (by induction hypothesis). Arranging the operators $\epsilon^{i}$ and $\eta^{i}$ back again in their original order we thus get

$$
\left(b \phi_{1}\right) \epsilon^{0}=b\left(\phi\left(\sigma \epsilon^{1}, \cdots, \sigma \epsilon^{n}\right) \epsilon^{0}\right)=b\left(\phi_{1} \epsilon^{0}\right) .
$$

Let $\psi=\sigma-\phi\left(\sigma \epsilon^{1}, \cdots, \sigma \epsilon^{n}\right)$. Then in view of Lemma $10.1 \psi \epsilon^{i}=0$ for $i \neq 0$. Hence 


$$
\begin{aligned}
(b \psi) \epsilon^{0} & =a^{-1}\left(\psi-\phi\left(\psi \epsilon^{1}, \cdots, \psi \epsilon^{n}\right)\right) \epsilon^{0}+\phi\left(b\left(\psi \epsilon^{1}\right), \cdots, b\left(\psi \epsilon^{n}\right)\right) \epsilon^{0} \\
& =a^{-1}\left(\psi \epsilon^{0}-0\right)+0=a^{-1}\left(\psi \epsilon^{0}\right) \\
& =a^{-1}\left(\psi \epsilon^{0}-\phi\left(\psi \epsilon^{0} \epsilon^{1}, \cdots, \psi \epsilon^{0} \epsilon^{n-1}\right)\right)+\phi\left(b\left(\psi \epsilon^{0} \epsilon^{1}\right), \cdots b\left(\psi \epsilon^{0} \epsilon^{n-1}\right)\right) \\
& =b\left(\psi \epsilon^{0}\right) .
\end{aligned}
$$

Hence

$$
\begin{aligned}
(b \sigma) \epsilon^{0} & =\left(b\left(\psi+\phi_{1}\right)\right) \epsilon^{0}=(b \psi) \epsilon^{0}+\left(b \phi_{1}\right) \epsilon^{0} \\
& =b\left(\psi \epsilon^{0}\right)+b\left(\phi_{1} \epsilon^{0}\right)=b\left(\left(\psi+\phi_{1}\right) \epsilon^{0}\right)=b\left(\sigma \epsilon^{0}\right) .
\end{aligned}
$$

We now show that $b$ commutes with all degeneracy operators in dimension $\leqq n$. Let $\tau \in G[n-1]$, then in view of the induction hypothesis

$$
\begin{array}{lr}
b\left(\tau \eta^{i} \epsilon^{j}\right)=b\left(\tau \epsilon^{j-1} \eta^{i}\right)=(b \tau) \epsilon^{j-1} \eta^{i}=(b \tau) \eta^{i} \epsilon^{j} & i<j-1 ; \\
b\left(\tau \eta^{i} \epsilon^{j}\right)=b \tau=(b \tau) \eta^{i} \epsilon^{j} & i=j-1, j ; \\
b\left(\tau \eta^{i} \epsilon^{j}\right)=b\left(\tau \epsilon^{j} \eta^{i-1}\right)=(b \tau) \epsilon^{j} \eta^{i-1}=(b \tau) \eta^{i} \epsilon^{j} & i>j .
\end{array}
$$

Consequently application of Lemma 10.2 yields for $0 \leqq i \leqq n$

$$
\begin{aligned}
b\left(\tau \eta^{i}\right) & =a^{-1}\left(\tau \eta^{i}-\phi\left(\tau \eta^{i} \epsilon^{1}, \cdots, \tau \eta^{i} \epsilon^{n}\right)\right)+\phi\left(b\left(\tau \eta^{i} \epsilon^{1}\right), \cdots, b\left(\tau \eta^{i} \epsilon^{n}\right)\right) \\
& \left.=a^{-1}\left(\tau \eta^{i}-\tau \eta^{i}\right)+\phi\left((b \tau) \eta^{i} \epsilon^{1}, \cdots,(b \tau) \eta^{i} \epsilon^{n}\right)\right)=(b \tau) \eta^{i} .
\end{aligned}
$$

In order to show that $b: G[n] \rightarrow H[n]$ is an isomorphism consider a simplex $\sigma \in G[n]$ such that $b \sigma=0$. Then $(b \sigma) \epsilon^{i}=b\left(\sigma \epsilon^{i}\right)=0$. By the induction hypothesis this implies $\sigma \epsilon^{i}=0$. Hence

$$
0=b \sigma=a^{-1}(\sigma-\phi(0, \cdots, 0))+\phi(0, \cdots, 0)=a^{-1} \sigma
$$

and because $a^{-1}$ is an isomorphism this implies $\sigma=0$ i.e. $b: G[n] \rightarrow H[n]$ is a monomorphism. Now let $\tau \in I I[n]$ and let

$$
\sigma_{i}=b^{-1}\left(\tau \epsilon^{i}\right), \quad \rho=a\left(\tau-\phi\left(\tau \epsilon^{1}, \cdots, \tau \epsilon^{n}\right)\right) .
$$

Then

$$
\begin{aligned}
\tau & =a^{-1} \rho+\phi\left(b \sigma_{1}, \cdots, b \sigma_{n}\right) \\
& =a^{-1}\left(\rho+\phi\left(\sigma_{1}, \cdots, \sigma_{n}\right)-\phi\left(\sigma_{1}, \cdots, \sigma_{n}\right)\right)+\phi\left(b \sigma_{1}, \cdots, b \sigma_{n}\right) \\
& =b\left(\rho+\phi\left(\sigma_{1}, \cdots, \sigma_{n}\right)\right),
\end{aligned}
$$

i.e. $b: G[n] \rightarrow I[[n]$ is also an epimorphism and is thus an isomorphism.

Naturality of $b$ is now easily verified.

Appendix

12. The c.s.s. free abelian group generated by a c.s.s. complex. Definc a functor

$$
F A: S \rightarrow i^{V}
$$


as follows. Let $K \in S$ be an object. Then $F A K[n]$ is the free abelian group with a generator $\bar{\sigma}$ for each $\sigma \in K[n]$ and $(F A K) \alpha$ is the homomorphism given by

$$
((F A K) \alpha) \bar{\sigma}=\overline{\sigma \alpha} .
$$

$F A K$ is called the c.s.s. free abelian group generated by $K$. For each c.s.s. map $f: K \rightarrow L$ a c.s.s. homomorphism $F A f: F A K \rightarrow F A L$ is defined by

$$
(F A f) \bar{\sigma}=\overline{f \sigma}
$$

The functor $F A$ is closely related to the functors $H^{v}(\Gamma, \quad)$ and $C_{N}$ (or equivalently $\otimes \Gamma)$. The relation is expressed by the following theorem.

THEOREM (12.1). There exists a natural equivalence

$$
d: F A(s) \rightarrow H^{v}\left(\Gamma, C_{N}(s)\right) .
$$

Proof. For each object $K \in S$ define a c.s.s. homomorphism

$$
d: F A K \rightarrow H^{v}\left(\Gamma, C_{N} K\right)
$$

by

$$
d \bar{\sigma}=C_{N} \phi_{\sigma}
$$

where $\phi_{\sigma}: \Delta[n] \rightarrow K$ is the unique map such that $\phi_{\sigma} \alpha=\sigma \alpha$ for all $\alpha \in \Delta[n]$. The naturality of $d$ then is obvious. It is also clear that $d$ is an isomorphism in dimension 0 . Now suppose it has already been proved that $d$ is an isomorphism in dimension $<n$. Then it is sufficient to show that $d$ is also an isomorphism in dimension $n$. Let $D$ (resp. E) be the subgroup of $F A K[n]$ (resp. $\left.H\left(\Gamma[n], C_{N} K\right)\right)$ generated by the degenerate simplices. Then Lemma (10.2) and the additivity of $\phi$ imply

$$
\rho \in D \text { (resp. E) if and only if } \rho=\phi\left(\rho \epsilon^{1}, \cdots, \rho \epsilon^{n}\right) .
$$

Combining this with the fact that $d$ is an isomorphism in dimension $n-1$ we get that $d: D \rightarrow E$ is an isomorphism. It is easily seen that $D$ is freely generated by those $\bar{\sigma}$ for which $\sigma$ is degenerate. Consequently $F A K[n]=D+\widetilde{D}$, where $\widetilde{D}$ is the free abelian group generated by elements $\bar{\sigma}-\phi\left(\bar{\sigma} \epsilon^{1}, \cdots, \bar{\sigma} \epsilon^{n}\right)$ where $\sigma$ is nondegenerate. Similarly we get $H\left(\Gamma[n], C_{N} K\right)=E+a^{-1}\left(C_{N} K\right)_{n}$. It now follows from

$$
\begin{aligned}
d\left(\bar{\sigma}-\phi\left(\bar{\sigma} \epsilon^{1}, \cdots, \bar{\sigma} \epsilon^{n}\right)\right)\left(C_{N} \epsilon_{n}\right) & =\left(C_{N} \phi_{\sigma}\right)\left(C_{N} \epsilon_{n}\right)-d\left(\phi\left(\ddot{\sigma} \epsilon^{1}, \cdots, \bar{\sigma} \epsilon^{n}\right)\right)\left(C_{N} \epsilon^{n}\right) \\
& =C_{N} \sigma+0=C_{N} \sigma
\end{aligned}
$$

where $C_{N} \sigma$ is the generator of $C_{N} K$, corresponding with the nondegenerate simplex $\sigma$, that $d: \widetilde{D} \rightarrow a^{-1}\left(C_{N} K\right)_{n}$ is also an isomorphism and so is therefore $d: F A K[n] \rightarrow H\left(\Gamma[n], C_{N} K\right)$.

Let $X$ be a topological space, let $K=I I^{v}(\Sigma, X)$ (see $\left.\$ 4\right)$ and let $\phi \in K[0]$. 
Let again

$$
h_{*}: \pi_{n}(K ; \phi) \rightarrow H_{n}\left(C_{N} K\right)
$$

be the Hurewicz homomorphism. Consider the (natural) map

$$
f: K \rightarrow F A K
$$

defined by $f \sigma=\bar{\sigma}, \sigma \in K$.

Proposition (12.2). Commutativity holds in the diagram

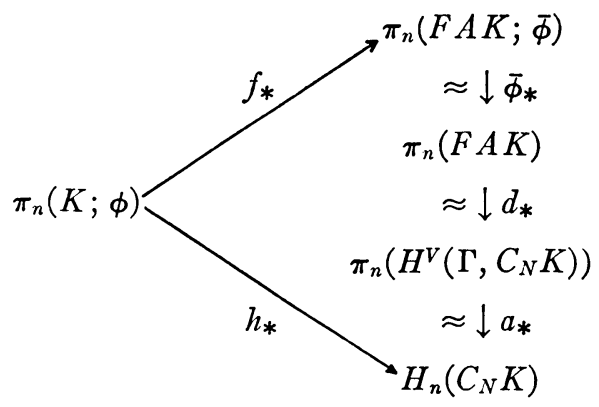

i.e. the map $f: K \rightarrow F A K$ induces (up to a natural equivalence) the Hurewicz homomorphisms.

Proof. Let $v \in \pi_{n}(K ; \phi), v \neq 0$ and let $\sigma \in v$. If $C_{N} \sigma$ is the corresponding generator of $C_{N} K$, then $C_{N} \sigma \in h_{*} v \in H_{n}\left(C_{N} K\right)$. Furthermore

$$
\begin{aligned}
\bar{\phi} \# f \sigma & =\bar{\sigma}-\bar{\phi} \eta^{0} \cdots \eta^{n-1}, \\
d \bar{\phi} f \sigma & =C_{N} \phi_{\sigma}-(d \bar{\phi}) \eta^{0} \cdots \eta^{n-1}, \\
a d \phi \# f \sigma & =\left(C_{N} \phi_{\sigma}\right)\left(C_{N} \epsilon_{n}\right)-\left((d \bar{\phi}) \eta^{0} \cdots \eta^{n-1}\right)\left(C_{N} \epsilon_{n}\right) \\
& =C_{N} \sigma-0=C_{N} \sigma .
\end{aligned}
$$

Hence

$$
a_{*} d_{*} \bar{\phi}_{*} f_{*}=h_{*} .
$$

\section{BiBLIOGRAPHY} 55-106.

1. S. Eilenberg and S. MacLane, On the groups $H(\pi, n)$, I, Ann. of Math. vol. 58 (1953) pp.

2. S. Eilenberg and J. A. Zilber, Semi-simplicial complexes and singular homology, Ann. of Math. vol. 51 (1950) pp. 499-513.

3. - On products of complexes, Amer. J. Math. vol. 75 (1953) pp. 200-204.

4. D. M. Kan, Adjoint functors, Trans. Amer. Math. Soc. vol. 87 (1958) pp. 294-329.

5. - On c.s.s. complexes, Amer. J. Math. vol. 79 (1957) pp. 449-476.

6. J. Milnor, The geometric realization of a semi-simplicial complex, Ann. of Math. vol. 65 (1957) pp. 357-362.

7. J. C. Moore, Algebraic homotopy theory, lecture notes, Princeton University, 1955-1956.

Columbia University,

NEW YoRK, N. Y. 